\title{
Meiotic recombination in Caenorhabditis elegans
}

\author{
Tatiana Garcia-Muse \& Simon J. Boulton* \\ DNA Damage Response Laboratory, Clare Hall Laboratories, Cancer Research UK, Blanch Lane, \\ South Mimms EN6 3LD, UK; Tel: +44 (0)1707 625 774; Fax: +44 (0)207 269 3801; \\ E-mail:simon.boulton@cancer.org.uk \\ * Correspondence
}

Key words: Caenorhabditis elegans, meiosis, recombination, synapsis, synaptonemal complex

\begin{abstract}
The faithful segregation of homologous chromosomes during meiosis is dependent on the formation of physical connections (chiasma) that form following reciprocal exchange of DNA molecules during meiotic recombination. Here we review the current knowledge in the Caenorhabditis elegans meiotic recombination field. We discuss recent developments that have improved our understanding of the crucial steps that must precede the initiation and propagation of meiotic recombination. We summarize the pathways that impact on meiotic prophase entry and the current understanding of how chromosomes reorganize and interact to promote homologous chromosome pairing and subsequent synapsis. We pay particular attention to the mechanisms that contribute to meiotic DNA double-strand break (DSB) formation and strand exchange processes, and how the C. elegans system compares with other model organisms. Finally, we highlight current and future areas of research that are likely to further our understanding of the meiotic recombination process.
\end{abstract}

\section{Caenorhabditis elegans: a model system to study meiotic prophase progression}

Meiosis is the specialized cell division by which diploid organisms generate haploid gametes key to sexual reproduction (Roeder 1997, Page \& Hawley 2003). The reduction in chromosome number in the gametes is achieved by one round of DNA replication followed by two divisions (known as meiosis I and meiosis II); in the first division, homologous chromosomes are segregated, followed by the segregation of sister chromatids in the next round of division corresponding to meiosis II. To achieve correct chromosome segregation during meiosis I, homologous chromosomes must first recognize each other and align along their lengths (Zickler \& Kleckner 1998). Once alignment and pairing is achieved synapsis occurs between homologous chromosomes with the assembly of a proteinaceous structure known as the synaptonemal complex (SC), which ensures that homologs remain in close proximity until the obligate crossover has been generated by double-strand break (DSB)-induced recombination (Roeder 1997, Zickler \& Kleckner 1999). It is within the context of the SC that crossover recombination occurs, which ultimately gives rise to crossovers that visibly manifest as chiasma. In addition to shuffling genes, crossover recombination is crucial because the chiasma is a physical link between homologs that enable them to correctly orient toward opposite spindle poles, an event that is critical for faithful segregation at the first meiotic division (Petronczki et al. 2003). Failure to correctly align chromosomes on the meiotic spindle frequently 
results in aneuploidy, which has catastrophic consequences for embryonic development (Hassold \& Hunt 2001).

C. elegans is a well-established model organism for the study of meiosis. The nematode is amenable to genetic manipulation through forward and reverse genetic approaches, which permits dissection of complex pathways. This system also possesses welldeveloped cytological tools, such as fluorescence in-situ hybridization (FISH) (Dernburg et al. 1998) and immunolocalization (Zetka et al. 1999), useful for meiotic mutant characterization. Meiosis in C. elegans not only displays canonical features with other eukaryotes but the linear progression through meiotic events within the gonad makes the adult hermaphrodite a particularly attractive model system to study meiosis (Albertson et al. 1997 C. elegans II). In the adult $C$. elegans hermaphrodite, germline nuclei in the distal tip of the gonad arm divide mitotically and thereafter enter meiotic prophase I as they progress away from the distal end of the gonad. The prophase of meiotic division I is traditionally divided into five sequential stages - leptotene, zygotene, pachytene, diplotene and diakinesis defined by a number of characteristic morphological changes associated with homolog alignment, pairing, synapsis and desynapsis. Direct visual observation of the hallmarks of each phase has been a useful starting point for the analysis of meiotic mutants in C. elegans (Figure 1). Following pre-meiotic S-phase, initial pairing events between homologous chromosomes takes place in the transition zone (Dernburg et al. 1998) when the polarized redistribution of chromosomes give rise to the characteristic 'crescent'-shaped DNA. In early pachytene, homolog synapsis resulting from formation of the SC can be recognized in nuclei as parallel DAPI-stained chromosomes that track either side of the SC (Goldstein \& Slaton 1982, Dernburg et al. 1998, MacQueen et al. 2002, Colaiacovo et al. 2003). Synapsis is visibly complete by mid-pachytene. Once the obligate crossover has formed desynapsis is initiated and chromosome condensation begins during diplotene (Nabeshima et al. 2005). It is at this stage that the emerging chiasmata can be first seen holding homologous chromosomes together. Finally, oocyte nuclei arrested at diakinesis can be recognized in the proximal region of the germline with six distinct DAPI-stained bodies or bivalents, that correspond to highly condensed homologous chromosome pairs attached by the chiasma. After fertilization, in the subsequent metaphase I, all the bivalents line up on the spindle, and at anaphase I the two duplicated homologs separate from each other and move to the opposite poles of the spindle, and the cell divides. To produce haploid gametes, a second cell division is required in which sister chromatids are segregated (McCarter et al. 1999).

A number of different genetic screens have been particularly useful in defining genes that impact on meiotic progression in $C$. elegans. The 'high incidence of males' or Him phenotype (Hodgking et al. 1979) is a powerful diagnostic of chromosome segregation defects. C. elegans hermaphrodites possess two X chromosomes (XX), while males are X0. Hermaphrodites produce both sperm and oocytes, and can reproduce either by self-fertilization or by mating with males, which only produce sperm. Males normally arise among the self-progeny of hermaphrodites at low frequency $(0.1-0.2 \%)$ through stochastic non-disjunction of the $\mathrm{X}$ chromosome. Mutations in many meiotic genes lead to errors in X chromosome segregation that manifest as a significant increase in the frequency of males. Frequently, the Him phenotype of meiotic mutants is coupled with extensive embryonic lethality, as a consequence of autosome non-disjunction. An improved screening method for meiotic mutants based on the Him phenotype has been to look for an increase in males produced by hermaphrodites carrying a Pxol-1::gfp reporter transgene (Kelly et al. 2000). The 'green eggs and Him' screen is based on the fact that Poxl$1: \because g f p$ transgene expresses only in male embryos. The presence of increased numbers of green-fluorescent embryos therefore correlates with the Him phenotype. Importantly, since many meiotic mutants are inviable, mutagenized hermaphrodites containing elevated numbers of GFP-positive embryos can be immediately crossed to wild-type males to facilitate rescue of lethal meiotic genes. This dual strategy has been very useful in identifying meiotic components required for pairing and recombination processes (see below).

\section{From mitosis to meiosis}

As mitotic cells move away from the distal end of the gonad they undergo pre-meiotic DNA replication as they enter the meiotic program. After chromosomes 


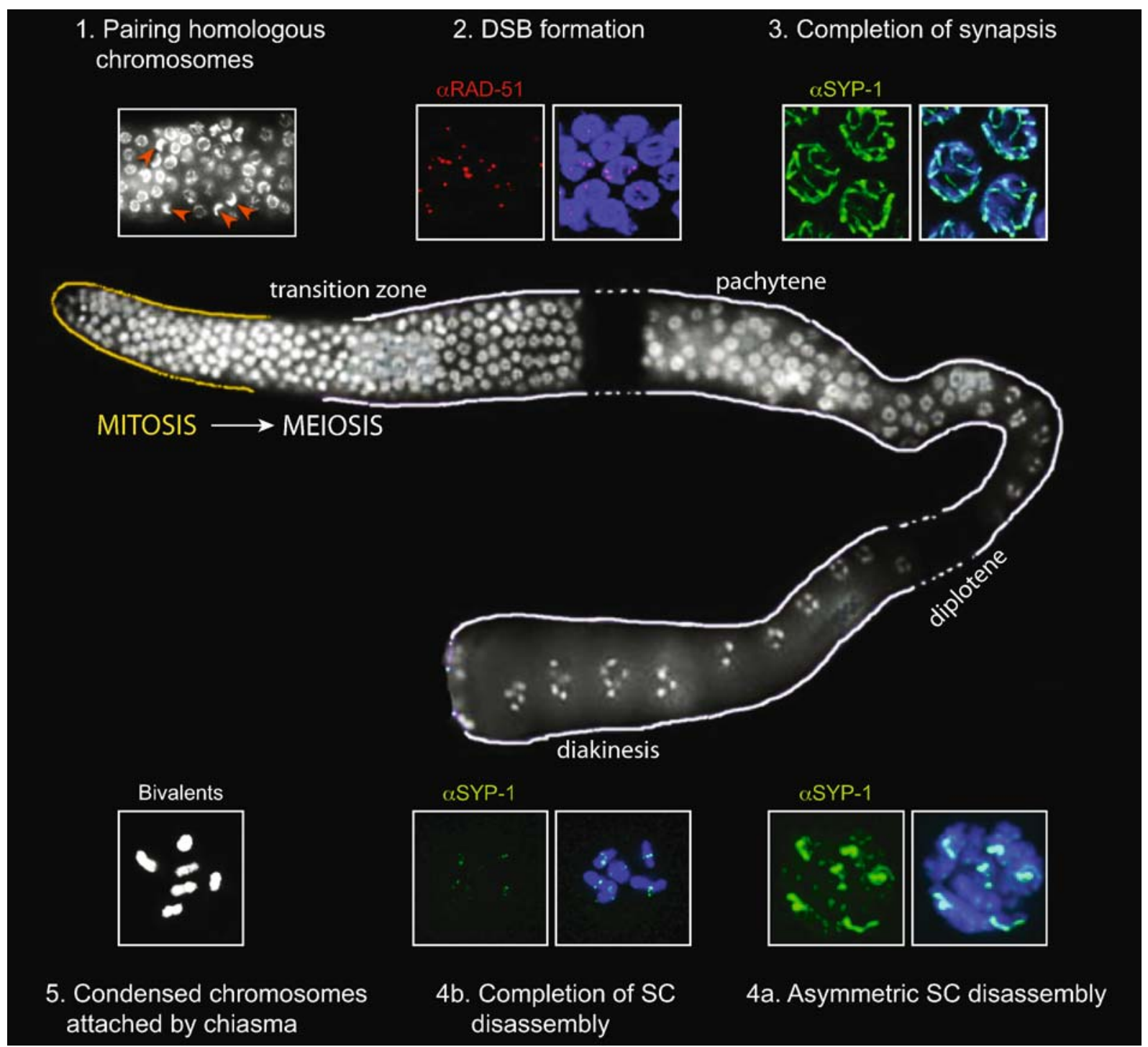

Figure 1. Schematic representation of the hallmarks of meiotic prophase progression in the C. elegans germline. Shown in a representative image of a DAPI-stained germline extracted from wild-type (N2) worms (modified from J. Maciejowski \& E.J. Hubbard), and immunostaining with RAD-51 and SYP-1 antibodies (MacQueen et al. 2002, Alpi et al. 2003).

have been duplicated during DNA replication, the sister chromatids remain tightly linked along their length by the loading of cohesin complexes (CohenFix 2001, Lee \& Orr-Weaver 2001). The cohesion of sister chromatids has been well defined within the context of the mitotic cell cycle, where sister chromatids are separated during a single division cycle through the proteolytic cleavage of the cohesin complexes by separase (Ciosk et al. 1998, Uhlmann et al. 1999). In contrast, the maintenance of cohesion between sister chromatids is essential for the correct segregation of homologous chromosomes at meiosis I; cohesion is first released along the arms at the onset of anaphase I but sister chromatids remain tethered by cohesin complexes retained in the vicinity of centromeres. These cohesin complexes remain until the second meiotic division. Meiotic cohesin complexes differ from their mitotic equivalents in that certain mitotic components (Scc1/Mdc1/Rad21, Scc3/SA1/SA2, Smc1 and Smc3) are replaced by 




Figure 2. Schematic of the sequential steps and the proteins involved during meiotic prophase progression in C. elegans. 
meiosis-specific variants. In yeast only Scc1 is replaced by meiosis-specific Rec8 (Klein et al. 1999, Watanabe \& Nurse 1999), whereas in mammalian meiosis two additional replacements occur: Smc1 by Smc1 $\beta$, and STAG3 for SA1/SA2 (Prieto et al. 2001, Revenkova et al. 2001).

While single candidates where found for SCC-3 (Wang et al. 2003) and the two subunits belonging to the structural maintenance of chromosomes (SMC) family (Pasierbek et al. 2003, Chan et al. 2003), SMC-1 (or HIM-1) and SMC-3, REC-8 is one of the four $\operatorname{Rad} 21 / \mathrm{Scc} 1 / \operatorname{Rec} 8$ homologs present in C. elegans genome (Pasierbek et al. 2001). REC-8 localizes at chromosomal axes throughout meiosis I, and depletion causes embryonic lethality, a strong Him phenotype and cytological defects associated with defective synapsis and premature separation of sister chromatids prior to the first meiotic division. Evidence suggests that REC- 8 is the meiotic cohesin protein homolog to $\operatorname{Rad} 21 / \operatorname{Rec} 8$. Reciprocal co-immunoprecipitation of SCC-3, SMC-1, SMC-3 and REC-8 indicates that these four proteins form a complex. Furthermore, SCC-3, SMC-1 and SMC-3 recapitulated the meiotic localization pattern described for REC- 8 and their depletion or deletion produced a phenotype with cytological defects analogous to that observed in the absence of rec- 8 (Chan et al. 2003). Taken together these data confirm that REC-8, SCC-3, SMC-1 and SMC-3 consitute the $C$. elegans meiotic cohesin complex required for the correct segregation of homologous chromosomes (Figure 2). Although sister chromatids are not held together in worms deficient for meiotic cohesin components, it has been shown that the initial recognition and pairing of homologs in leptotene/zygotene is only decreased. This suggests that the initial homolog interactions leading to pairing are not solely dependent on cohension and SC formation.

\section{Finding your partner: homolog alignment and pairing}

During pairing, homologous chromosomes recognize each other, align and become physically connected along their entire length. How homologous chromosomes recognize each other is still uncertain. For many organisms, models involving complementary DNA base-pair interactions between intact duplex at numerous and widely dispersed sites along chromosomes have been proposed (Weiner \& Kleckner 1994, Gerton \& Hawley 2005). A number of elegant studies have established that $C$. elegans chromosomes possess specialized pairing regions that are required for stabilizing homolog interactions and for promoting initiation of synapsis. Evidence for such sites came from experiments examining the meiotic behavior of strains carrying various chromosome rearrangements/deletions that led to the identification of cis-acting regions on each chromosome which are collectively termed pairing centers (PC) (McKim et al. 1993, Villeneuve 1994 , MacQueen et al. 2005).

Two recent studies have characterized four related zinc-finger proteins (HIM-8, ZIM-1, ZIM-2 and ZIM-3), which directly interact with the PC and are essential for promoting pairing and homologous chromosome synapsis during meiosis (Phillips et al. 2005, Phillips \& Dernburg 2006). Analysis revealed that HIM-8 and ZIM-2 interact with the PC on chromosome $\mathrm{X}$ and $\mathrm{V}$ respectively, ZIM-1 associates with the PC on chromosomes II and III, and ZIM-3 interacts with the PC on chromosomes I and IV (Figure 2). It is currently unclear why the $\mathrm{X}$ and $\mathrm{V}$ chromosome have unique $\mathrm{PC}$ binding proteins or why the two remaining pairs of autosomes share a single protein. This indicates that the specificity of homolog recognition cannot be solely defined by either the role of the $\mathrm{PC}$ or by the identity of the corresponding ZIM/HIM-8 family member. It is also interesting to note that the PC-associated protein ZIM/HIM-8 associates with the nuclear envelope and that, in the case of the three ZIM, their subnuclear localization seems to be lost in chk-2 mutants (Phillips \& Dernburg 2006) that are compromised for homolog alignment and pairing (MacQueen et al. 2001).

CHK-2 is a member of the checkpoint kinase Cds1/Rad53 family (MacQueen \& Villeneuve 2001, Oishi et al. 2001). Analysis of the chk-2 mutant revealed that CHK-2 is required for the initial establishment of pairing between homologous chromosomes as well as for chiasma formation. Indeed, chk-2 mutants fail to exhibit the characteristic crescent-shaped nuclear morphology associated with homolog pairing. The pairing defect in $c h k-2$ mutants can now be partially explained by the mislocalization of the ZIM proteins. However, HIM-8 localization to the $\mathrm{X}$ chromosome $\mathrm{PC}$ is unaffected in the chk-2 mutants, implying that the regulation of pairing 
differs between autosomes and the $\mathrm{X}$ chromosome (Phillips \& Dernburg 2006). Despite this, the precise role for $\mathrm{CHK}-2$ in controlling homolog pairing remains to be elucidated. An intriguing possibility is that phosphorylation of ZIM proteins by CHK-2 might be important for their correct subcellular localization. The possibility that phosphorylation events might be more generally involved in controlling the various stages of meiotic prophase progression has not been explored in any great detail. However, studies are currently under way to identify all potential phosphorylation sites in known $C$. elegans meiotic proteins using peptide array technologies. Peptide arrays can be used in conjunction with specific recombinant kinases such as CHK-2 or more generally with $C$. elegans meiotic extracts. Coupling this in-vitro approach with in-vivo assays, such as mutant complementation, will be important for confirming the relevance of specific phosphorylation events in controlling meiotic prophase progression. This kind of analysis has the potential to further our understanding of the regulation of meiosis in C. elegans.

\section{Synapsis through SC formation}

In organisms such as yeast and mammals, initiation of meiotic recombination is a prerequisite to SC formation. In contrast, SC formation occurs independent of initiation of meiotic recombination in $C$. elegans and Drosophila melanogaster (reviewed by Gerton \& Hawley 2005, Roeder 1997). Indeed, C. elegans mutants in genes required for initiation or completion of meiotic recombination assemble the SC as normal (Dernburg et al. 1998, Alpi et al. 2003). The lack of chiasmata between homologs only becomes apparent cytologically in these mutants as the SC disassembles (see below). The lack of a requirement of DSB to initiate synapsis likely reflects the use of alternative ways of homolog recognition such as the use of pairing centers in the worm and somatic pairing in the fly (MacQueen et al. 2005, Vazquez et al. 2002). In all organisms, however, the recombination process is completed while homologous chromosomes are held in close juxtaposition by the SC, which likely aids in homology searching prior to strand exchange and heteroduplex formation.

The SC is a highly ordered tripartite structure composed of two parallel lateral elements, corresponding to the proteinaceous scaffolds along the individual chromosomes, and a central region, which contains transverse elements that lie perpendicular to the lateral elements (reviewed by Heyting 1996, Page \& Hawley 2004). The axial elements play important roles in chromosome condensation, pairing, synapsis, and inhibiting meiotic recombination between sister chromatids; while the central element forms the basis of synapsis and is likely involved in crossover interference (see below). In yeast, DNA is organized around the lateral element, which contains the Rec8 cohesin and axial elements such Red1 and Hop1 (Hollingsworth et al. 1990, Hollingsworth \& Johnson 1993). No obvious Red1 homologs have been described in mammals or $C$. elegans, although functional counterparts of Hop1 do exist. Hop1 belongs to the meiosis-specific HORMA domain protein family (mammals: HORMAD1; C. elegans: HIM-3, HTP-1, HTP-2 and HTP-3). So far all the $C$. elegans HORMA proteins have been implicated in structural maintenance and dynamics of homolog pairing and synapsis (Zetka et al. 1999, Couteau et al. 2004, Couteau \& Zetka 2005, Martinez-Perez \& Villeneuve 2005, MacQueen et al. 2005). HIM-3 is a major component of the lateral element, and localizes to chromosomes prior to synapsis where it co-localizes with REC-8 along the chromosome length (Figure 2). Analysis of him-3 mutants indicates that HIM-3 facilitates synapsis by promoting the polymerization of the SC central core elements: SYP-1 and SYP-2 (Zetka et al. 1999, Colaiacovo et al. 2003, Couteau et al. 2004). It has also been proposed that HIM-3 might perform a role in enforcing recombination between homologs whilst acting as a barrier to repair through the sister, analogous to Hop1, its ortholog in budding yeast (Niu et al. 2005).

In $C$. elegans meiotic recombination can be monitored by analyzing the dynamics of assembly and disassembly of the strand exchange protein RAD-51 onto meiotic DSB (Alpi et al. 2003, Martin et al. 2005). In the absence of synapsis meiotic DSBs are not efficiently repaired and RAD-51 foci persist, presumably because the homolog is not in close juxtaposition, and repair through the sister is temporarily blocked. Indeed, in the him-3 null mutant homologous chromosomes fail to synapse, yet the kinetics of assembly and disassembly of RAD-51 foci at meiotic DSB resembles that of the wild type (Zetka et al. 1999, Colaiacovo et al. 2003, Couteau 
et al. 2004). This suggests that, although DSBs cannot be repaired through the homolog (due to the defect in synapsis), they are repaired with normal kinetics through the sister chromatid. It is therefore likely that HIM-3 normally functions to temporarily prevent DSB repair between sister chromatids to ensure formation of the obligate crossover between homolog pairs, analogous to its yeast counterpart Hop1 (Niu et al. 2005).

A similar defect in using the sister chromatid as a template for meiotic DSB repair is observable in htp-1 mutants, although HTP-1 differs from HIM-3 in that it also prevents the stabilization of inappropriate synapsis between nonhomologous autosomes (Couteau \& Zetka 2005, Martinez-Perez \& Villeneuve 2005). Exactly how HTP-1 functions to prevent inappropriate synapsis remains unclear. Currently, conclusive evidence for the function of HTP-2 in meiosis has been hampered by the lack of a null mutant for $h t p-2$, although RNAi supports a role in promoting SC assembly (Couteau \& Zetka 2005). Studies in yeast indicate that Mnd1-Hop2 complex is a meiosis-specific heterodimer required to ensure and promote strand invasion with the correct template DNA (Tsubouchi \& Roeder 2002, Pezza et al. 2006). It therefore appears that the functions of HTP-1 and HTP-2 resemble that of Mnd1-Hop2 in S. cerevisiae, although there is no obvious sequence similarity between the $C$. elegans and yeast proteins.

SYP-1 and SYP-2 are components of the central region of the $\mathrm{SC}$ in $C$. elegans (MacQueen et al. 2002, Colaiacovo et al. 2003). Although there is no obvious sequence similarity among SC core components between different species, these proteins contain a central coiled-coil motif, that is also present in the yeast homolog Zip1 and in mammalian SCP1, which are both required for normal meiotic recombination, respectively (Meuwissen et al. 1992, Sym et al. 1993). In the absence of SYP-1 or SYP-2 meiotic DSBs are created with normal timing, as revealed by the timely accumulation of RAD-51 foci, but synapsis fails to occur. However, RAD-51 foci persist throughout pachytene and only begin to disappear in diplotene nuclei, presumably following repair of meiotic DSB between sister chromatids, once the barrier to such events has been removed (Colaiacovo et al. 2003). The presence of 12 intact univalents at diakinesis in syp mutants suggests that meiotic DSB repair has occurred. This situation contrasts with the highly irregular decondensed chro- mosomes evident at diakinesis in mutants defective for meiotic DSB repair (e.g. rad-51 or brc-2 mutants (Rinaldo et al. 2002, Martin et al. 2005)).

It is known that the yeast SC central region components Zip2 and Zip3 are both required to promote Zip1 assembly between homologous chromosomes (Chua \& Roeder 1998, Agarwal \& Roeder 2000). It is believed that Zip3 marks the DSB sites that will become a crossover and subsequently recruits Zip2 to finally promote SC formation via Zip1 assembly (Agarwal \& Roeder 2000). Although yeast and $C$. elegans differ in their dependence on DSB formation for synapsis a sequence homolog for Zip3 is present in C. elegans (ZPH-3 (Jantsch et al. 2004)). Contrary to its 'homolog' in yeast, chromosome localization of ZHP-3 is dependent on SYP-1 but independent of DSB formation. Deletion of zhp-3 does not affect SC or DSB formation, but the presence of univalents at diakinesis indicates that this protein is essential for meiotic recombination at some level (Jantsch et al. 2004). The precise function of ZHP-3 in $C$. elegans meiotic recombination remains unclear.

\section{Control of meiotic DSB formation}

Crossover or chiasma formation requires temporal coordination of the initiation of meiotic recombination with progression through prophase, but molecular events ensuring this coordination remain elusive. Meiotic recombination is initiated in all eukaryotes by the formation of a DSB through the action of a specialized topoisomerase enzyme Spo11 (Keeney et al. 1997). In C. elegans, spo-11 mutants exhibit extensive embryonic lethality and a severe Him phenotype, indicative of errors in meiotic chromosome segregation (Dernburg et al. 1998). Cytological analysis of spo-11 mutants reveals 12 univalents at diakinesis, instead of the six bivalents in wild-type animals, suggestive of a defect in crossover formation. Consistent with the proposed role for SPO-11 in DSB generation, spo- 11 mutants are partially rescued by artificially inducing DSBs using ionizing radiation, as was shown for the yeast spoll mutant (Figure 2). Furthermore, RAD-51 foci fail to form in spo-11 mutants, indicative of an absence of DSBs. Although the mechanism for initiation of meiotic recombination is conserved throughout eukaryotes, the analysis of spo- 11 mutants revealed that pairing and synapsis 
occur normally (Dernburg et al. 1998). The fact that SC formation occurs independent of spo- 11 in $C$. elegans permits the analysis of mutants that function in the recombination process per se without the added complication that defects observed may result from failure to initiate synapsis.

In addition to Spo11 nine other genes are known to be absolutely required for meiotic DSB formation in yeast, i.e. Rad50, Mre11, Xrs2, Mer2, Mei4, Rec102, Rec104, Rec114 and Ski8. Rad50, Mre11 and Xrs2 encode a conserved protein complex critical for Spo11 function, DSB processing and sensing of DSBs in mitotic cells (see below) (Gerton \& Hawley 2005). Several observations suggest that Spo11 acts together with Ski8, Rec102 and Rec104 in a multiprotein complex essential for DSB formation (Arora et al. 2004, Kee et al. 2004); Ski8 (a WD repeats protein) acts as scaffold protein for Spo11 localization to chromatin and to Rec102/Rec104 complex, whose role is still unclear. No Rec102 or Rec104 sequence homologs are found in C. elegans or mammals. Two recent studies suggest that Mer2, Mei4 and Rec114 also form a complex (Henderson et al. 2006, Li et al. 2006). Moreover, Mer2 is a substrate for the yeast cyclin-dependent kinase Cdc28, suggestive of regulatory connection between cell cycle and meiotic DSB formation (Henderson et al. 2006). Phosphorylation of Mer2 appears to modulate its interactions with the Rec114/Mei4 complex but more studies are necessary to clarify the exact function of this complex. Again no sequence homologs have been found in C. elegans or mammalians for these three proteins. The absence of homologs in C. elegans could be explained by an alternative mechanism of DSB initiation and regulation, due to the different scenarios in which DSB formation is initiated: along paired chromosomes in yeast and mostly within the context of the SC in worms. But this would not explain why the search for homologs in mammalian genomes has been unsuccessful. A more likely scenario is that functional homologs exist in all eukaryotes but are not obvious based on sequence homology alone. It is likely that further analysis of the yeast DSB-initiation complexes may suggest opportunities for identification of functional equivalents in higher eukaryotes.

It is been speculated that the yeast meiotic DSB initiation complexes may somehow affect competence for DSB formation through inducing alterations in chromosome structure, potentially through epigenetic modifications. In this respect a recently characterized protein, HIM-17, is the first reported factor in $C$. elegans that functions to establish competence for DSB formation through inducing modifications in chromatin (Reddy \& Villeneuve 2004). him-17 mutants exhibit 12 univalents at diakinesis, are defect for RAD-51 focus formation and, like the spo- 11 mutant, both phenotypes can be partially rescued by introducing artificial DSB using ionizing radiation. These data place HIM-17 function at the level of meiotic DSB formation. HIM-17 was shown to localize to chromatin throughout the germline, suggestive of a role on chromatin. Indeed, immunostaining of him-17 mutants with antibodies to lysine 9 methylated histone H3 (H3MeK9), a marker of heterochromatin and transcriptionally inactive DNA, showed an abnormal pattern (Reddy $\&$ Villeneuve 2004). These results suggested that HIM-17 might induce changes to chromatin structure that are important for SPO-11 function, potential at the level of chromatin accessibility. To understand how the chromatin modifications induced by HIM-17 regulate meiotic DBS formation is clearly an important area of investigation that may shed further light on the regulation of SPO-11 action.

Following the generation of a DSB by Spo11 the DNA ends at the break site must be first processed into $3^{\prime}$ single-stranded DNA tails by the combined action of helicases and exonucleases. The MRN complex (consisting of MRE11, RAD50, and NBS1/Xrs2) is required for DSB formation, processing, and checkpoint signaling during meiotic cell division in yeast (van den Bosch et al. 2003). C. elegans possesses homologs for Mre11 and Rad50 (Chin \& Villeneuve 2001, Colaiacovo et al. 2002) and a putative candidate that shares weak similarity to part of the human NBS1 protein. Consistent with a critical meiotic function mre-11 mutants exhibit 12 univalents at diakinesis indicative of a defect in meiotic recombination. Unlike spo-11 mutants, IR-treatment of mre-11 mutants does not restore crossover formation but instead leads to chromosomal abnormalities at diakinesis. The latter is suggestive of a role in DSB processing but it is currently unclear if meiotic DSB are actually formed in mre-11 mutants, but are not efficiently processed, or if MRE-11 is required for the generation of DSBs by SPO-11. RNAi knockdown of rad-50 has been shown to result in univalents at diakinesis analogous to spo- 11 and mre- 11 mutants but confirmation of the 
precise meiotic function of rad-50 will require studies in a deletion mutant.

\section{Molecular events of meiotic recombination}

In all organisms the RecA/Rad51 family of recombinases catalyze the strand-invasion and strandexchange reactions between homologous DNA molecules (West 2003). Homologs and paralogs have been found in all eukaryotes; among these, Dmc1, which encodes the meiotic ortholog of budding yeast Rad51 (Bishop et al. 1992). In C. elegans there is only one recombinase protein RAD-51, which acts in both mitotic and meiotic recombination (Rinaldo et al. 2002). The processed ssDNA tails at DSBs are the substrate onto which monomers of RAD-51 polymerize to form a nucleoprotein filament, which can be visualized cytologically as discrete nuclear foci that form in a SPO-11-dependent manner at meiotic DSB. The RAD-51-DNA filament executes the central functions in homologous recombination: search for the homologous template and invasion into an intact homologous sequence with the formation of heteroduplex DNA (Figure 2). Although bacterial RecA is a potent recombinase, its eukarytotic counterparts (Rad51), including C. elegans RAD-51 (Petalcorin et al. 2006) are weak recombinase enzymes that require additional co-factors/mediators to potentiate their activity in recombination. Yeast $\operatorname{Rad} 52$ is the prototypical mediator that binds directly to Rad51, facilitates its loading onto DSBs, and stimulates Rad51-mediated strand exchange and D-loop formation. Surprisingly, C. elegans does not possess a Rad52 homolog, a situation that is mirrored in D. melanogaster and Arabidopsis thaliana. Although Rad52 is critical to recombination in yeast, the mammalian homolog is largely dispensable for recombination. Rather it appears that alternative mediator proteins have taken over the role of $\operatorname{Rad} 52$ in facilitating Rad51 recombination reactions. Indeed central to recombination in higher eukaryotes is BRCA2, one of the two major breast and ovarian cancer tumor-suppressor proteins.

The human BRCA2 protein contains eight BRC repeats that confer direct binding to Rad51 and three oligonucleotide-oligosaccharide binding (OB-fold) domains that mediate binding to ssDNA/dsDNA transitions (Tutt et al. 2001, Venkitaraman 2000, 2001). Based on these observations, and the fact that cells lacking BRCA2 fail to load RAD51 onto DNA breaks, it has been speculated that BRCA2 recruits RAD51 to sites of DNA damage where it may facilitate binding of RAD51 onto processed breaks and could modulate other RAD51 activities (Patel et al. 1998, Jasin 2002, Moynahan 2002, Moynahan et al. 2001, Wilson \& Elledge 2002). How this occurs at the mechanistic level is not well understood. Since human BRCA2 is a 3418 aa protein, it has not been possible to express and purify the full-length protein for biochemical analysis.

One of the hallmarks of BRCA2 is the BRC motif. In a search for proteins with similarities to the BRC, a putative homolog of BRCA2 was identified in $C$. elegans (CeBRC-2) (Martin et al. 2005). Remarkably, this protein possesses a single BRC and one OB-fold condensed into a 394aa polypeptide. CeBRC-2 was shown to interact directly with RAD-51 in vitro and in vivo through its single BRC domain (Martin et al. 2005). The single OB-fold situated in the C-terminal part of CeBRC-2 confers preferential binding to ssDNA. CeBRC-2 also contains two consensus nuclear localization signals (NLS) that flank the OB-fold domain. It is noticeable that RAD-51 lacks an obvious NLS, analogous to its human counterpart. Indeed, CAPAN1 cells that harbor a truncating mutation in human BRCA2 exhibit cytoplasmic localization of Rad51, indicating that one of the normal functions of BRCA2 is to facilitate transport of Rad51 into the nucleus. A very similar situation exists in C. elegans; Cebrc-2 mutants are defect for efficient nuclear localization of RAD-51. Although CeBRC-2 is approximately one-tenth the size of its human counterpart, deletion mutants in Cebrc-2 exhibit many other defects associated with $B R C A 2$ deficiency in human cells, indicating that the critical functions of BRCA2 are conserved in C. elegans. Specifically, CeBRC-2 prevents extensive chromosomal fragmentation during early embryogenesis and is required for the repair of both meiotic and IR-induced DSB, defects that are partially attributable to a failure to target Rad51 into the nucleus. In addition to a role in nuclear localization of RAD-51, dominant negative experiments using the BRC domain of CeBRC-2 alone revealed a role in facilitating loading of RAD-51 onto processed meiotic DSB. Unlike rad-51 mutants, animals deficient for CeBRC-2 accumulate replication protein $\mathrm{A}$ (RPA) foci that are dependent on meiotic DSB formation by SPO-11 (Martin et al. 
2005). These data suggested that, in the absence of CeBRC-2, DSBs are hyper-resected to generate extensive regions of ssDNA. The fact that CeBRC-2 is recruited to meiotic DSBs in the absence of RAD-51, and that rad-51 mutants do not accumulate RPA foci at meiotic DSB, suggests that CeBRC-2 is able to load onto DSB independent of RAD-51, displace RPA from ssDNA and likely minimizes extensive DSB processing by exonucleases. However, its normal function in wild-type cells is to facilitate nuclear localization and loading of RAD-51 onto meiotic DSB (Figure 2).

Unlike spo-11 and mre-11 mutants, animals deficient for rad-51 or Cebrc-2 exhibit extensive chromosomal abnormalities and chromatin decondensation at diakinesis (Martin et al. 2005). This difference from the situation observed in spo- 11 and mre- 11 mutants likely reflects the role for RAD-51 and CeBRC-2 in all aspects of meiotic DSB repair; crossover and non-crossover formation and repair of DSB using the sister chromatid as a template. The fact that mre- 11 mutants resemble spo-11 mutants, but not rad-51 or Cebrc-2, also supports the idea that MRE-11 is required for meiotic DSB formation, although this requires further confirmation. Surprisingly, analysis of Cebrc-2 mutants also suggested functions independent of $\mathrm{rad}-51$ in an error-prone repair pathway distinct from NHEJ (Martin et al. 2005). It was speculated that, since $C$. elegans lack a Rad52 homolog, CeBRC-2 may have taken over the role of $\operatorname{Rad} 52$ in regulating RAD-51 during HR and may also function in errorprone repair via the single strand-annealing pathway (SSA).

Biochemical analysis supports a role for CeBRC-2 in facilitating RAD-51 functions post formation of the RAD-51 nucleoprotein filament. It was demonstrated that recombinant CeBRC-2 stimulated RAD51-mediated D-loop formation and reduced the rate of ATP hydrolysis catalyzed by RAD-51 (Petalcorin et al. 2006). These findings, together with related studies using Ustilago maydis BRCA2 and RAD51 proteins and fragments of human BRCA2, suggested that CeBRC-2 and its counterparts in other eukaryotes function as mediators of RAD-51-dependent recombination reactions (Kojic et al. 2002, Yang et al. 2002, 2005). The fact that CeBRC-2 also inhibits ATP hydrolysis by RAD-51 would suggest that CeBRC-2 can prevent RAD-51 nucleoprotein filament depolymerization; ATP hydrolysis leads to depolymerization of the RAD-51-DNA filament (Galletto et al. 2006, Joo et al. 2006).

Human BRCA2 also associates with a small, highly acidic protein DSS1 that is strongly conserved throughout eukaryotes (Marston et al. 1999, Yang et al. 2002). DSS1 appears to play an important role in the HR pathway as disruption of Dss1 in $U$. maydis confers meiotic recombination defects that are epistatic with the Rad51 pathway. Furthermore, siRNA of DSS1 in human cells leads to the accumulation of abnormal DNA structures and a defect in Rad51 focus formation at DNA damage sites, analogous to the defects observed in BRCA2deficient cells (Kojic et al. 2003, Gudmundsdottir et al. 2004). The C. elegans DSS1 ortholog (Y119D3B.15) possesses $75 \%$ sequence identity to its human and U. maydis counterparts. However, the helical domain-OB1 region that binds DSS1 in human BRCA2 is not obviously conserved in CeBRC-2. Although DSS-1 significantly improves the solubility of CeBRC-2 when the two proteins are co-expressed in E. coli (Petalcorin et al. 2006), DSS-1 and CeBRC-2 do not co-purify over gel filtration and do not interact in either yeast two-hybrid or in pulldown experiments. Moreover, DSS-1 is dispensable for CeBRC-2 to stimulate RAD-51-mediated D-loop formation (Petalcorin et al. 2006). A deletion mutant in $d s s-1$ has recently become available that will hopefully provide insight into any potential functions in meiotic recombination.

A number of other proteins implicated in meiotic recombination in yeast and/or mammalian cells are also present in $C$. elegans. These include homologs of BRCA1, BARD1, a Rad51 paralog, and Rad54 (BRC-1, BRD-1, RFS-1 and RAD-54, respectively). It appears that BRC-1 and its heterodimeric partner BRD-1 do not play critical roles in meiotic recombination as deletion mutants exhibit six intact bivalents at diakinesis indicative of successful crossover recombination (Boulton et al. 2004). However, both mutants exhibit a weak Him phenotype that may suggest a subtle role in recombination. The precise function of BRC-1/BRD-1 in meiosis is currently unclear but this is an area that we are actively working on. Although deletion mutants in $r f s-1$ and rad-54 are available meiotic recombination functions have yet to be reported (Figure 2). Understanding how these accessory proteins interplay with Rad51 is critical to dissect the mechanisms of meiotic 
recombination. What remains completely unclear is how RAD-51 dependent homology searching and subsequent strand invasion events are performed within the context of the SC. It is unlikely that the SC simply acts as a scaffold, but may actively facilitate all steps required for initiation, propagation and completion of meiotic recombination.

The processing of strand exchange intermediates produces recombinant products that have either exchanged the flanking DNA arms (crossovers) or have not undergone exchange (non-crossovers). Crossover outcome in C. elegans is dependent on the meiosis-specific members of the MutS family, HIM-14/MSH-4 and MSH-5, conserved components of the core meiotic recombination machinery that promote crossover outcomes of initiated recombination events (Zalevsky et al. 1999, Kelly et al. 2000, Colaiacovo et al. 2003). Cytological analysis of $m s h$ 4/him-14 and msh-5 mutants revealed that homologs are paired and aligned and meiotic DSBs are generated as indicated by the formation of RAD-51 foci (Colaiacovo et al. 2003). However, the presence of persistent RAD-51 foci and 12 univalents in these mutants suggests that HIM-14/MSH-4 and MSH-5 perform a critical role after the formation of the RAD-51 nucleoprotein filament but upstream of Holliday junction resolution. Although msh-4/ him-14 and msh-5 encode members of the MutS family no difference in spontaneous mutation frequency or defects in IR-induced DSB repair has been detected (Kelly et al. 2000). This suggests that HIM-14/MSH-4 and MSH-5, like their orthologs in budding yeast, have no detectable role in mismatch repair but rather may function specifically to promote crossing over during meiosis. In contrast to yeast, where mutants that lack Msh4 and/or Msh5 can still generate $30-50 \%$ of the normal crossovers (RossMacdonald \& Roeder 1994, Hollingsworth et al. 1995), C. elegans relies exclusively on this pathway to generate crossovers, since the lack of either gene eliminates all crossovers. Although genetic studies have shown that MSH4-MSH5 strongly influences crossover formation their mechanism of action remains unclear. Markers that indicate the position of crossovers are not currently available in C. elegans. However, MLH1, a commonly used marker of crossovers in certain organisms (Baker et al. 1996), is present in $C$. elegans but remains uncharacterized (Figure 2).
The precise mechanisms that regulate crossingover remain poorly understood in all organisms. Since crossover formation is critical for accurate chromosome segregation at the first meiotic division specific mechanisms must exist in all organisms to ensure that at least one crossover is generated per homolog pair. Crossover interference, a phenomenon in which the generation of a crossover at a particular site somehow interferes with the coincident occurrence of another crossover, results in crossovers that are widely spaced along the chromosome length (van Veen \& Hawley 2003, Bishop \& Zickler 2004). In the majority of higher eukaryotes one to three crossovers are formed per homolog pair. However, studies in $C$. elegans have revealed that one crossover is formed per bivalent, suggesting that a single crossover is capable of suppressing the formation of a second crossing over event along the entire chromosome length; this is an extreme case of crossover interference (Hillers \& Villeneuve 2003). Support for a role for the chromosome axis in crossover interference has come from analysis of a hypomorphic allele in the axial component him-3, which retains the capacity to synapse and undergo crossover formation despite altered chromosome axis composition (Nabeshima et al. 2004). The analysis of this mutant provided evidence that the integrity of chromosome axis plays an important role in limiting the number of crossovers per homologous chromosome pair as an increase in double crossovers was observed. It should be noted that this mutant exhibits increased RAD-51 foci, raising the possibility that the increase in double crossover events may result from up-regulation in DSB formation. Understanding the mechanism of crossover interference is one of the outstanding challenges in the meiosis field. $C$. elegans is clearly an intriguing system for studying this phenomenon given the restriction of a single crossover event per homolog pair.

Evidence suggests that the chromosomal location of the crossover is also tightly controlled in $C$. elegans. The distribution of sites of meiotic exchanges on autosomes is restricted to regions that lie outside of gene clusters located in the middle of the chromosome; crossing over tends to occur in the $30 \%$ of the chromosome length from each autosome end (Brenner 1974, Hillers \& Villeneuve 2003). Curiously the sites of meiotic exchanges on the $\mathrm{X}$ chromosome differ from autosomes as they are more 
evenly distributed along the chromosome. Genetic studies have identified an uncloned locus, rec-1, that alters the distribution of crossovers on autosomes resulting in an increase in the frequency of crossovers within the central gene cluster region (Zetka \& Rose 1990, 1995). It is therefore likely that the cloning of the mutation responsible for rec-l will provide important insights into the control of crossover formation.

Completion of meiotic recombination requires the resolution of heteroduplex structures that may resemble Holliday junctions. The prototypical resolvase in $E$. coli is RuvABC that enzymatically cleaves Holliday junctions in a symmetrical manner (reviewed in West 1997). Despite the absence of sequence homologs of RuvABC in higher eukaryotes mammalian cells do possess a similar resolvase activity associated with the Rad51C paralog, but the exact components of this enzymatic complex remain elusive (Liu et al. 2004). Evidence from fission yeast also suggests that structure-specific endonucleases such as Mus81 play important roles in completing meiotic recombination (Boddy et al. 2001, Chen et al. 2001, Kaliraman et al. 2001). Whilst homologs of Rad51 paralogs, Mus81 and XPF are present in $C$. elegans the endonuclease responsible for completing meiotic recombination is not yet known (Figure 2).

\section{Summary}

During the past decade $C$. elegans has developed as a powerful system for studying meiotic prophase progression. It is clear that the factors that have made $C$. elegans an attractive system for such studies (forward and reverse genetics, spatial organization of the germline, uncoupled meiotic DSB formation and synapsis, etc.) will continue to aid our goal of understanding the genes and pathways that impact on meiosis. The recent identification of chromosome pairing centres and their associated Zn-finger binding

Table 1. List of the known C. elegans proteins required for the indicated steps in meiosis I in comparison with yeast and mammalian counterparts. Asterisks indicate the putative homologs identified by sequence homology, which remain to be characterized.

\begin{tabular}{|c|c|c|c|}
\hline Function & S. cerevisiae & Mammals & C. elegans \\
\hline PCs & $?$ & $?$ & HIM-8 \& ZIMs \\
\hline cohesion & $\begin{array}{l}\text { Rec8p } \\
\text { Scc3 } \\
\text { Smc1 } \\
\text { Smc3 }\end{array}$ & $\begin{array}{l}\text { REC8 } \\
\text { STAG3 } \\
\text { SMC1ß } \\
\text { SMC3 }\end{array}$ & $\begin{array}{l}\text { REC-8 } \\
\text { SCC-3 } \\
\text { SMC-1 } \\
\text { SMC-3 }\end{array}$ \\
\hline $\mathrm{SC}$ & $\begin{array}{l}\text { Red1 - Hop1 } \\
\text { Mnd1-Hop2 } \\
? \\
\text { Zip1p } \\
\text { Zip3 Zip2 }\end{array}$ & $\begin{array}{l}\text { HORMAD1* } \\
\text { MND1- ? } \\
? \\
\text { SCP1 } \\
\text { SCP2 SCP3 }\end{array}$ & $\begin{array}{l}\text { ? HIM-3 } \\
\text { HTP-1 - HTP-2 } \\
\text { HTP-3 } \\
\text { SYP-1 SYP-2 } \\
\text { ZHP-3 ? }\end{array}$ \\
\hline DSB formation & $\begin{array}{l}\text { Spo11p } \\
\text { Ski8 - Rec102 - Rec104 } \\
\text { Mer2 - Mei4 - Rec114 } \\
\text { Mre11 Rad50 Xrs2 }\end{array}$ & $\begin{array}{l}\text { SPO11 } \\
? ? ? \\
? ? ? \\
\text { MRE11 RAD50 NSB1 }\end{array}$ & $\begin{array}{l}\text { SPO-11 } \\
? ? ? \\
? ? ? \\
\text { MRE-11 RAD-50 XNP-1* }\end{array}$ \\
\hline Meiotic recombination & $\begin{array}{l}\text { Rad51 } \\
\text { Dmc1 } \\
\text { Rad55, Rad57 } \\
\text { Mei5-Sae3 } \\
\text { Rad52? } \\
\text { Rad54 } \\
\text { Msh4-Msh5 } \\
\text { Mlh1 } \\
\text { Mus81? }\end{array}$ & $\begin{array}{l}\text { RAD51 } \\
\text { DMC1 } \\
\text { RAD51B-C-D, XCCC2-3 } \\
\text { ? ? } \\
\text { (RAD52?) BRCA2 } \\
\text { RAD54 } \\
\text { MSH4 MSH5 } \\
\text { MLH1 } \\
\text { EME1 } \\
\text { MUS81 }\end{array}$ & $\begin{array}{l}\text { RAD-51 } \\
\text { RAD-51 } \\
\text { RFS-1* } \\
\text { ? ? } \\
\text { ? BRC-2 } \\
\text { RAD-54* } \\
\text { MSH-4/HIM-14 MSH-5 } \\
\text { MLH-1* } \\
\text { EME-1* } \\
\text { MUS-81* }\end{array}$ \\
\hline
\end{tabular}


factors has placed $C$. elegans at the forefront of meiotic research into homolog recognition, pairing and synapsis. Analogous to the mitotic cell cycle, meiotic progression is likely to be controlled by a plethora of post-translational modifications of which we know very little. The demonstrations that the CHK-2 kinase is critical for homolog alignment and pairing and HIM-17 is important for meiotic DSB formation likely represent the first of many factors that impact on meiotic progression through posttranslational modification. Future studies in these areas are likely to advance our understanding of key steps in meiosis in all organisms (Table 1).

In the years ahead C. elegans is likely to contribute significantly to our understanding of conserved mechanisms of meiotic prophase progression, such as initiation of meiotic DSB formation and synapsis, whilst mechanistic differences with other models will bring a better understanding of how complex issues can be solved by alternative means.

\section{References}

Agarwal S, Roeder GS (2000) Zip3 provides a link between recombination enzymes and synaptonemal complex proteins. Cell 102: 245-255.

Albertson DG, Rose AM, Villeneuve AM (1997). Chromosome Organization, Mitosis and Meiosis. C. Elegans II. Cold Spring Harbor: Cold Spring Harbor Laboratory Press.

Alpi A, Pasierbek P, Gartner A, Loidl J (2003) Genetic and cytological characterization of the recombination protein RAD51 in Caenorhabditis elegans. Chromosoma 112: 6-16.

Arora C, Kee K, Maleki S, Keeney S (2004) Antiviral protein Ski8 is a direct partner of Spo11 in meiotic DNA break formation, independent of its cytoplasmic role in RNA metabolism. Mol Cell 13: 549-559.

Baker SM, Plug AW, Prolla TA et al. (1996) Involvement of mouse Mlh1 in DNA mismatch repair and meiotic crossing over. Nat Genet 13: 336-342.

Bishop DK, Park D, XU L, Kleckner N (1992) DMC1: a meiosisspecific yeast homolog of $E$. coli recA required for recombination, synaptonemal complex formation, and cell cycle progression. Cell 69: 439-456.

Bishop DK, Zickler D (2004) Early decision: meiotic crossover interference prior to stable strand exchange and synapsis. Cell 117: $9-15$.

Boddy MN, Gaillard PH, McDonald WH, Shanahan P, Yates JR III, Russell P (2001) Mus81-Eme1 are essential components of a Holliday junction resolvase. Cell 107: 537-548.

Boulton SJ, Martin JS, Polanowska J, Hill DE, Gartner A, Vidal M (2004) BRCA1/BARD1 orthologs required for DNA repair in Caenorhabditis elegans. Curr Biol 14: 33-39.
Brenner S (1974) The genetics of Caenorhabditis elegans. Genetics 77: 71-94.

Chan RC, Chan A, Jeon M et al. (2003) Chromosome cohesion is regulated by a clock gene paralogue TIM-1. Nature 423: 1002-1009.

Chen XB, Melchionna R, Denis CM et al. (2001) Human Mus81associated endonuclease cleaves Holliday junctions in vitro. Mol Cell 8: 1117-1127.

Chin GM, Villeneuve AM (2001) C. elegans mre-11 is required for meiotic recombination and DNA repair but is dispensable for the meiotic G(2) DNA damage checkpoint. Genes Dev 15: 522-534.

Chua PR, Roeder GS (1998) Zip2, a meiosis-specific protein required for the initiation of chromosome synapsis. Cell $\mathbf{9 3}$ : 349-359.

Ciosk R, Zachariae W, Michaelis C, Shevchenko A, Mann M, Nasmyth K (1998) An ESP1/PDS1 complex regulates loss of sister chromatid cohesion at the metaphase to anaphase transition in yeast. Cell 93: 1067-1076.

Cohen-Fix O (2001) The making and breaking of sister chromatid cohesion. Cell 106: 137-140.

Colaiacovo MP, MacQueen AJ, Martinez-Perez E et al. (2003) Synaptonemal complex assembly in C. elegans is dispensable for loading strand-exchange proteins but critical for proper completion of recombination. Dev Cell 5: 463-474.

Colaiacovo MP, Stanfield GM, Reddy KC, Reinke V, Kim SK, Villeneuve AM (2002) A targeted RNAi screen for genes involved in chromosome morphogenesis and nuclear organization in the Caenorhabditis elegans germline. Genetics 162: 113-128.

Couteau F, Nabeshima K, Villeneuve A, Zetka M (2004) A component of $C$. elegans meiotic chromosome axes at the interface of homolog alignment, synapsis, nuclear reorganization and recombination. Curr Biol 14: 585-592.

Couteau F, Zetka M (2005) HTP-1 coordinates synaptonemal complex assembly with homolog alignment during meiosis in C. elegans. Genes Dev 19: 2744-2756.

Dernburg AF, McDonald K, Moulder G, Barstead R, Dresser M, Villeneuve AM (1998) Meiotic recombination in C. elegans initiates by a conserved mechanism and is dispensable for homologous chromosome synapsis. Cell 94: 387-398.

Galletto R, Amitani I, Baskin RJ, Kowalczykowski SC (2006) Direct observation of individual RecA filaments assembling on single DNA molecules. Nature 443: 875-878.

Gerton JL, Hawley RS (2005) Homologous chromosome interactions in meiosis: diversity amidst conservation. Nat Rev Genet 6: $477-487$.

Goldstein P, Slaton DE (1982) The synaptonemal complexes of Caenorhabditis elegans: comparison of wild-type and mutant strains and pachytene karyotype analysis of wild-type. Chromosoma 84: 585-597.

Gudmundsdottir K, Lord CJ, Witt E, Tutt AN, Ashworth A (2004) DSS1 is required for RAD51 focus formation and genomic stability in mammalian cells. EMBO Rep 5: 989-993.

Hassold T, Hunt P (2001) To err (meiotically) is human: the genesis of human aneuploidy. Nat Rev Genet 2: 280-291.

Henderson KA, Kee K, Maleki S, Santini PA, Keeney S (2006) Cyclin-dependent kinase directly regulates initiation of meiotic recombination. Cell 125: 1321-1332. 
Heyting C (1996) Synaptonemal complexes: structure and function. Curr Opin Cell Biol 8: 389-396.

Hillers KJ, Villeneuve AM (2003) Chromosome-wide control of meiotic crossing over in C. elegans. Curr Biol 13: 16411647.

Hodgking J, Horvitz HR, Brenner S (1979) Nondisjunction mutants of the nematode Caenorhabditis elegans. Genetics $\mathbf{9 1}$ 64-94.

Hollingsworth NM, Goetsch L, Byers B (1990) The HOP1 encodes a meiosis-specific component of yeast chromosomes. Cell $\mathbf{6 1}$ : 73-84.

Hollingsworth NM, Johnson AD (1993) A conditional allele of the Saccharomyces cerevisiae HOP1 gene is suppressed by overexpression of two other meiosis-specific genes: RED1 and REC104. Genetics 133: 785-797.

Hollingsworth NM, Ponte L, Halsey C (1995) MSH5, a novel MutS homolog, facilitates meiotic reciprocal recombination between homologs in Saccharomyces cerevisiae but not mismatch repair. Genes Dev 9: 1728-1739.

Jantsch V, Pasierbek P, Mueller MM, Schweizer D, Jantsch M, Loidl J (2004) Targeted gene knockout reveals a role in meiotic recombination for ZHP-3, a Zip3-related protein in Caenorhabditis elegans. Mol Cell Biol 24: 7998-8006.

Jasin M (2002) Homologous repair of DNA damage and tumorigenesis: the BRCA connection. Oncogene 21: 8981-8993.

Joo C, McKinney SA, Nakamura M, Rasnik I, Myong S, Ha T (2006) Real-time observation of RecA filament dynamics with single monomer resolution. Cell 126: 515-527.

Kaliraman V, Mullen JR, Fricke WM, Bastin-Shanower SA, Brill SJ (2001) Functional overlap between Sgs1-Top3 and the Mms4-Mus81 endonuclease. Genes Dev 15: 2730-2740.

Kee K, Protacio RU, Arora C, Keeney S (2004) Spatial organization and dynamics of the association of Rec102 and Rec104 with meiotic chromosomes. EMBO J 23: 1815-1824.

Keeney S, Giroux CN, Kleckner N (1997) Meiosis-specific DNA double-strand breaks are catalyzed by Spo11, a member of a widely conserved protein family. Cell 88: 375-384.

Kelly KO, Dernburg AF, Stanfield GM, Villeneuve AM (2000) Caenorhabditis elegans msh-5 is required for both normal and radiation-induced meiotic crossing over but not for completion of meiosis. Genetics 156: 617-630.

Klein F, Mahr P, Galova M et al. (1999) A central role for cohesins in sister chromatid cohesion, formation of axial elements, and recombination during yeast meiosis. Cell $\mathbf{9 8}$ : 91-103.

Kojic M, Kostrub CF, Buchman AR, Holloman WK (2002) BRCA2 homolog required for proficiency in DNA repair, recombination, and genome stability in Ustilago maydis. Mol Cell 10: 683-691.

Kojic M, Yang H, Kostrub CF, Pavletich NP, Holloman WK (2003) The BRCA2-interacting protein DSS1 is vital for DNA repair, recombination, and genome stability in Ustilago maydis. Mol Cell 12: 1043-1049.

Lee JY, Orr-Weaver TL (2001) The molecular basis of sisterchromatid cohesion. Annu Rev Cell Dev Biol 17: 753-777.

Li J, Hooker GW, Roeder GS (2006) Saccharomyces cerevisiae Mer2, Mei4 and Rec114 form a complex required for meiotic double-strand break formation. Genetics 173: 1969-1981.
Liu Y, Masson JY, Shah R, O’Regan P, West SC (2004) RAD51C is required for Holliday junction processing in mammalian cells. Science 303: 243-246.

MacQueen AJ, Villeneuve AM (2001) Nuclear reorganization and homologous chromosome pairing during meiotic prophase require C. elegans chk-2. Genes Dev 15: 1674-1687.

MacQueen AJ, Colaiacovo MP, McDonald K, Villeneuve AM (2002) Synapsis-dependent and -independent mechanisms stabilize homolog pairing during meiotic prophase in C. elegans. Genes Dev 16: 2428-2442.

MacQueen AJ, Phillips CM, Bhalla N, Weiser P, Villeneuve AM, Dernburg AF (2005) Chromosome sites play dual roles to establish homologous synapsis during meiosis in C. elegans. Cell 123: 1037-1050.

Marston NJ, Richards WJ, Hughes D, Bertwistle D, Marshall CJ, Ashworth A (1999) Interaction between the product of the breast cancer susceptibility gene BRCA2 and DSS1, a protein functionally conserved from yeast to mammals. Mol Cell Biol 19: 4633-4642.

Martin JS, Winkelmann N, Petalcorin MI, McIlwraith MJ, Boulton SJ (2005) RAD-51-dependent and -independent roles of a Caenorhabditis elegans BRCA2-related protein during DNA double-strand break repair. Mol Cell Biol 25: 3127-3139.

Martinez-Perez E, Villeneuve AM (2005) HTP-1-dependent constraints coordinate homolog pairing and synapsis and promote chiasma formation during C. elegans meiosis. Genes Dev 19: 2727-2743.

Meuwissen RL, Offenberg HH, Dietrich AJ, Riesewijk A, Van Iersel M, Heyting C (1992) A coiled-coil related protein specific for synapsed regions of meiotic prophase chromosomes. EMBO J 11: 5091-5100.

McCarter J, Bartlett B, Dang T, Schedl T (1999) On the control of oocyte meiotic maturation and ovulation in Caenorhabditis elegans. Dev Biol 205: 111-128.

McKim KS, Peters K, Rose AM (1993) Two types of sites required for meiotic chromosome pairing in Caenorhabditis elegans. Genetics 134: 749-768.

Moynahan ME (2002) The cancer connection: BRCA1 and BRCA2 tumor suppression in mice and humans. Oncogene 21: 8994-9007.

Moynahan ME, Pierce AJ, Jasin M (2001) BRCA2 is required for homology-directed repair of chromosomal breaks. Mol Cell 7: 263-272.

Nabeshima K, Villeneuve AM, Colaiacovo MP (2005) Crossing over is coupled to late meiotic prophase bivalent differentiation through asymmetric disassembly of the SC. J Cell Biol 168: 683-689.

Nabeshima K, Villeneuve AM, Hillers KJ (2004) Chromosomewide regulation of meiotic crossover formation in Caenorhabditis elegans requires properly assembled chromosome axes. Genetics 168: 1275-1292.

Niu H, Wan L, Baumgartner B, Schaefer D, Loidl J, Hollingsworth NM (2005) Partner choice during meiosis is regulated by Hop1-promoted dimerization of Mek1. Mol Biol Cell 16: 5804-5818.

Oishi I, Iwai K, Kagohashi Y et al. (2001) Critical role of Caenorhabditis elegans homologs of Cds1 (Chk2)-related kinases in meiotic recombination. Mol Cell Biol 21: 1329-1335. 
Page SL, Hawley RS (2003) Chromosome choreography: the meiotic ballet. Science 301: 785-789.

Page SL, Hawley RS (2004) The genetics and molecular biology of the synaptonemal complex. Annu Rev Cell Dev Biol 20: 525-552.

Pasierbek P, Fodermayr M, Jantsch V, Jantsch M, Schweizer D, Loidl J (2003) The Caenorhabditis elegans SCC-3 homologue is required for meiotic synapsis and for proper chromosome disjunction in mitosis and meiosis. Exp Cell Res 289: 245-255.

Pasierbek P, Jantsch M, Melcher M, Schleiffer A, Schweizer D, Loidl J (2001) A Caenorhabditis elegans cohesion protein with functions in meiotic chromosome pairing and disjunction. Genes Dev 15: 1349-1360.

Patel KJ, Yu VP, Lee H et al. (1998) Involvement of Brca2 in DNA repair. Mol Cell 1: 347-357.

Petalcorin MI, Sandall J, Wigley DB, Boulton SJ (2006) CeBRC-2 stimulates D-loop formation by RAD-51 and promotes DNA single-strand annealing. J Mol Biol 361: 231-242.

Petronczki M, Siomos MF, Nasmyth K (2003) Un menage a quatre: the molecular biology of chromosome segregation in meiosis. Cell 112: 423-440.

Pezza RJ, Petukhova GV, Ghirlando R, Camerini-Otero RD (2006) Molecular activities of meiosis-specific proteins Hop2, Mnd1, and the Hop2-Mnd1 complex. J Biol Chem 281: 18426-18434.

Phillips CM, Dernburg AF (2006) A family of zinc-finger proteins is required for chromosome-specific pairing and synapsis during meiosis in C. elegans. Dev Cell 11: 817-829.

Phillips CM, Wong C, Bhalla N et al. (2005) HIM-8 binds to the $\mathrm{X}$ chromosome pairing center and mediates chromosome-specific meiotic synapsis. Cell 123: 1051-1063.

Prieto I, Suja JA, Pezzi N et al. (2001) Mammalian STAG3 is a cohesin specific to sister chromatid arms in meiosis I. Nat Cell Biol 3: 761-766.

Reddy KC, Villeneuve AM (2004) C. elegans HIM-17 links chromatin modification and competence for initiation of meiotic recombination. Cell 118: 439-452.

Revenkova E, Eijpe M, Heyting C, Gross B, Jessberger R (2001) Novel meiosis-specific isoform of mammalian SMC1. Mol Cell Biol 21: 6984-6998.

Rinaldo C, Bazzicalupo P, Ederle S, Hilliard M, La Volpe A (2002) Roles for Caenorhabditis elegans rad-51 in meiosis and in resistance to ionizing radiation during development. Genetics 160: 471-479.

Roeder GS (1997) Meiotic chromosomes: it takes two to tango. Genes Dev 11: 2600-2621.

Ross-MacDonald P, Roeder GS (1994) Mutation of a meiosisspecific MutS homolog decreases crossing over but not mismatch correction. Cell 79: 1069-1080.

Sym M, Engebrecht JA, Roeder GS (1993) ZIP1 is a synaptonemal complex protein required for meiotic chromosome synapsis. Cell 72: 365-378.

Tsubouchi H, Roeder GS (2002) The Mnd1 protein forms a complex with hop2 to promote homologous chromosome pairing and meiotic double-strand break repair. Mol Cell Biol 22: 3078-3088.

Tutt A, Bertwistle D, Valentine J et al. (2001) Mutation in Brca2 stimulates error-prone homology-directed repair of DNA double-strand breaks occurring between repeated sequences. EMBO J 20: 4704-4716.
Uhlmann F, Lottspeich F, Nasmyth K (1999) Sister-chromatid separation at anaphase onset is promoted by cleavage of the cohesin subunit Scc1. Nature 400: 37-42.

van den Bosch M, Bree RT, Lowndes NF (2003) The MRN complex: coordinating and mediating the response to broken chromosomes. EMBO Rep 4: 844-849.

van Veen JE, Hawley RS (2003) Meiosis: when even two is a crowd. Curr Biol 13: R831-R833.

Vazquez J, Belmont AS, Sedat JW (2002) The dynamics of homologous chromosome pairing during male Drosophila meiosis. Curr Biol 12: 1473-1483.

Venkitaraman AR (2000) The breast cancer susceptibility gene, BRCA2: at the crossroads between DNA replication and recombination? Phil Trans $R$ Soc Lond B Biol Sci 355: 191-198.

Venkitaraman AR (2001) Functions of BRCA1 and BRCA2 in the biological response to DNA damage. J Cell Sci 114: 3591-3598.

Villeneuve AM (1994) A cis-acting locus that promotes crossing over between $\mathrm{X}$ chromosomes in Caenorhabditis elegans. Genetics 136: 887-902.

Wang F, Yoder J, Antoshechkin I, Han M (2003) Caenorhabditis elegans EVL-14/PDS-5 and SCC-3 are essential for sister chromatid cohesion in meiosis and mitosis. Mol Cell Biol 23: 7698-7707.

Watanabe Y, Nurse P (1999) Cohesin Rec8 is required for reductional chromosome segregation at meiosis. Nature 400: 461-464.

Weiner BM, Kleckner N (1994) Chromosome pairing via multiple interstitial interactions before and during meiosis in yeast. Cell 77: 977-991.

West SC (1997) Processing of recombination intermediates by the RuvABC proteins. Annu Rev Genet 31: 213-244.

West SC (2003) Molecular views of recombination proteins and their control. Nat Rev Mol Cell Biol 4: 435-445.

Wilson JH, Elledge SJ (2002) Cancer: BRCA2 enters the fray. Science 297: 1822-1823.

Yang H, Jeffrey PD, Miller J et al. (2002) BRCA2 function in DNA binding and recombination from a BRCA2-DSS1-ssDNA structure. Science 297: 1837-1848.

Yang H, Li Q, Fan J, Holloman WK, Pavletich NP (2005) The BRCA2 homologue Brh2 nucleates RAD51 filament formation at a dsDNA-ssDNA junction. Nature 433: 653-657.

Zalevsky J, MacQueen AJ, Duffy JB, Kemphues KJ, Villeneuve AM (1999) Crossing over during Caenorhabditis elegans meiosis requires a conserved MutS-based pathway that is partially dispensable in budding yeast. Genetics 153: 1271-1283.

Zetka MC, Kawasaki I, Strome S, Muller F (1999) Synapsis and chiasma formation in Caenorhabditis elegans require HIM-3, a meiotic chromosome core component that functions in chromosome segregation. Genes Dev 13: 2258-2270.

Zetka MC, Rose AM (1990) Sex-related differences in crossing over in Caenorhabditis elegans. Genetics 126: 355-363.

Zetka MC, Rose AM (1995) Mutant rec-1 eliminates the meiotic pattern of crossing over in Caenorhabditis elegans. Genetics 141: 1339-1349.

Zickler D, Kleckner N (1998) The leptotene-zygotene transition of meiosis. Annu Rev Genet 32: 619-697.

Zickler D, Kleckner N (1999) Meiotic chromosomes: integrating structure and function. Annu Rev Genet 33: 603-754. 